\title{
Risk factors for breast cancer in Egyptian postmenopausal
}

\author{
females in Alexandria
}

Taha Ismail Hewala*, Nadia Ahmad Abd El-Moneim**, Gehan Shehata;***

\begin{abstract}
Background: Obesity is a modifiable risk factor for breast cancer in postmenopausal females. Aim: This study was conducted to investigate the association between obesity and breast cancer risk among Egyptian postmenopausal females in Alexandria. Subjects: This study included 100 Egyptian postmenopausal females, 50 of them were breast cancer patients with mean age (54.6 years \pm 9.6) and the other 50 were controls with mean age (53.9 years \pm 2.5$)$. Methods: Standardized questionnaires concerning age, anthropometric, menstrual and reproductive information, family history of breast cancer in first degree relatives and history of hypertension were completed by the same researcher. Fasting blood samples were withdrawn from all participating females to separate sera in which total estradiol, insulin and glucose levels were measured. Results: Univariate analysis showed that age at presentation $\geq 60$ years, delayed menopause $>50$ years, family history of breast cancer in first degree relatives, oral contraceptive use, body mass index (BMI), waist circumference (WC) and serum levels of total estradiol were found to be significantly associated with higher risk of breast cancer in postmenopausal females. Multivariate logistic regression analysis showed that old age at presentation $\geq 60$ years and late menopause $>50$ years were the strongest risk factors, while oral contraceptive use and increased WC $>100 \mathrm{~cm}$ showed border line significance. Conclusion: Based on the results obtained from this study, we advice Egyptian postmenopausal females of Alexandria to control weight by decreasing dietary caloric intake, maintain physical activity and breast self-examination continuously in order to decrease the risk of breast carcinoma.
\end{abstract}

Key words: Breast cancer, Obesity, estradiol, Insulin, Estrogen receptor, Progesterone receptor, postmenopausal females

\section{INTRODUCTION}

Breast cancer is one of the most decreases sensitivity of target tissues to

important problems of public health. ${ }^{(1)}$ circulating insulin resulting in

Postmenopausal obesity was found to hyperinsulinemia. ${ }^{(2)}$ Hyperinsulinemia leads

be a breast cancer risk factor. One of the to reduction in the level of circulating sex

consequences of obesity, insulin resistance hormone binding globulin (SHBG) coupled

\footnotetext{
*Departments of Radiation Sciences, Medical Research Institute, Alexandria University, Egypt.

${ }^{* *}$ Cancer Management and Research, Medical Research Institute, Alexandria University, Egypt.

***Biomedical Informatics and Medical Statistics. Medical Research Institute, Alexandria University, Egypt.
} 
with increased rate of proliferation of breast epithelial cells due to the increased amounts of free estradiol. ${ }^{(3)}$

Epidemiological analysis of breast cancer by hormone receptor status (HRS) shows distinct patterns for estrogen receptor positive $(E R+)$ and estrogen receptor negative (ER-) cancers. Risk factor distribution differs among patients based on HRS with reproductive factors that increase a woman's lifetime exposure to endogenous estrogens resulting in ER+ cancer. ${ }^{(4)}$ Other risk factors such as genetic risks, radiation and smoking give rise to ER- cancers. ${ }^{(5)}$ The importance of studying risk factors for breast cancer according to HRS originates from the fact that presence of hormone receptor positive $(\mathrm{HR}+)$ breast cancer implies the best response to antiestrogen therapy, whereas hormone receptor negative (HR-) breast cancer implies poor response to anti-estrogen therapy. ${ }^{(6)}$

Studies that investigated the association between HRS and obesity have yielded conflicting results. (7-10) Dey et al. ${ }^{(11)}$ conducted a study in Tanta, Egypt and observed that the incidence of ER+ breast cancers was 2-4 times higher in Urban than in rural areas. Based on the results of this study, the present study was conducted to shed light on the situation in Alexandria as an urban region in Egypt regarding the association between obesity, reproductive and menstrual factors and the risk of breast cancer according to estrogen and progesterone receptor.

\section{Subjects and Methods}

\section{Sample size calculation:}

Sample size was calculated using NCSS 2000 (Number Cruncher Statistical System) and PASS (Power Analysis and Sample Size) Program. The minimum sample size required was 100 (50 for each group) to achieve $80 \%$ power to detect a difference of 5 between both groups with estimated group standard deviations of 7.9 and 9.7 and with a significance level (alpha) of 0.05 using a two- 
sided sample t-test. ${ }^{(12)}$

\section{Subjects}

This study was conducted on 100 postmenopausal females admitted to the Department of Cancer Management \& Research of the Medical Research Institute, Alexandria University, Egypt, within the period from January to November 2010. A female was considered postmenopausal if more than 12 months passed since her last menstrual period. Females were divided into two groups: Group I (Breast cancer group): 50 postmenopausal females affected by histologically- and mammography-confirmed breast cancer, recently detected, not undergoing surgery and not receiving chemotherapy, of mean age (54.6 \pm 9.6 years). Group II (control group): 50 normal healthy postmenopausal females' volunteers with no breast pathology, of matched age (53.9 \pm 2.5 years) and socioeconomic status with patients of group I.

Exclusion criteria: included subjects who received medicine, dietary regimen or surgery to manage obesity within the last 12 months.

\section{METHODS}

Three tools were used to obtain relevant information: interview schedule, anthropometric measurements and laboratory investigations.

\section{1- Interview schedule}

After having approval from the ethical committee, Medical Research Institute, signed informed consents were obtained from all subjects who agreed to participate in this study. An interview schedule was designed to collect relevant information from all eligible females enrolled in this study. The schedule included the following items:

- Demographic information including age at presentation, age at menarche, age at menopause and age at first fullterm pregnancy.

- Personal data on obstetric history including: number of abortions and parity. 
- Anthropometric information including $\mathrm{BMI}$ and waist circumference.

- Months of breast feeding (cumulative number of months of breast feeding for all children) and history of oral contraceptive pills intake before menopause.

- Family history of breast cancer in first degree relatives.

- Personal history of hypertension.

\section{2- Anthropometric measurements}

Body mass index (BMI) was calculated as: $\mathrm{BMI}=$ Weight $(\mathrm{kg}) /$ square height (meter). Waist Circumference (WC) was measured with a tape measure mid-way between the lower rib margin and the iliac crest.

\section{3- Laboratory tests}

All laboratory tests were carried out at Radiation Sciences Department, Medical Research Institute, Alexandria University. Fasting blood samples were collected from controls and from patients before surgery. Immediately after withdrawing, blood samples were allowed to coagulate and centrifuged for 20 minutes at $3500 \mathrm{rpm}$ at 4 ${ }^{\circ} \mathrm{C}$. The separated serum samples were aliquoted, frozen at $-80^{\circ} \mathrm{C}$, and stored until assayed. After thawing, each serum aliquot was assayed only once. Fasting serum glucose level was measured using an enzymatic colorimetric method based on Trinder-reaction. ${ }^{(13)}$ Serum insulin levels were measured using a ready-for-use, commercially available double antibody radioimmunoassay (RIA) kit (Linco Research, USA) according to the manufacturer's protocol. Briefly, $100 \mu \mathrm{l}$ of each serum and $100 \mu$ of human insulin antibody were mixed by vortex, covered and incubated overnight at room temperature. $100 \mu \mathrm{l}$ of hydrated ${ }^{125} \mathrm{I}-$ radiolabeled insulin tracer were added, mixed by vortex, covered and incubated overnight at room temperature. $1.0 \mathrm{ml}$ of cold $\left(4^{\circ} \mathrm{C}\right)$ precipitating reagent (Goat antiGuinea Pig IgG Serum, 3\% PEG and $0.05 \%$ Triton $\mathrm{X}-100$ in $0.05 \mathrm{M}$ Phosphosaline, $0.025 \mathrm{M}$ EDTA, $0.08 \%$ 
sodium azide) was added, mixed by vortex and incubated for 20 minutes at $4^{\circ} \mathrm{C}$. The reaction mixture was centrifuged at $4^{\circ} \mathrm{C}$ for 20 minutes at 5,000 rpm. The supernatant was completely decanted and the pellet was counted in a gamma counter for 1 minute (perkin Elmer, Finland). A calibration curve was constructed using known different concentrations of recombinant human insulin to determine the serum levels of human insulin. The assay sensitivity was $0.2 \mu \mathrm{U} / \mathrm{ml}$ and the specificity was $100 \%$. Insulin resistance (IR) was quantified using the homeostatic model assessment (HOMA) according to the following equation ${ }^{(14)}$ :

HOMA-IR $=($ Glucose $(\mathrm{mg} / \mathrm{dl}) \times$ Insulin $(\mu \mathrm{U} / \mathrm{ml})) / 405$

Serum total estradiol (E2) levels were determined using a ready-for-use RIA kit (SIEMENS, USA) according to the manufacturer's protocol. Briefly, $100 \mu \mathrm{l}$ of serum and $1.0 \mathrm{ml}$ of ${ }^{125}$ I-radiolabeled E2 tracer were added to a polypropylene tube coated with E2 antibody. The mixture was mixed by vortex and incubated for 3 hours at room temperature. After incubation, reaction mixture was decanted thoroughly and the tube was counted for 1.0 minute in a gamma counter (perkin Elmer, Finland).

A calibration curve was constructed using known different concentrations of human E2 to determine the serum levels of human E2. The assay sensitivity was $8 \mathrm{pg} / \mathrm{ml}$. Data about ER and PR status were collected from patrents' pathology records after surgery.

\section{Statistical analysis:}

Data analysis was carried out using SPSS version 17, it included the following:

\section{A- Data processing}

Data processing had two major objectives namely clean data by performing a series of comprehensive checks and producing analytic results which involved the recoding of variables.

i. Range checking and skip checking: Range checking was performed to ensure 
that all questions had valid codes, whereas skip checking was done to ensure that the correct set of questions was answered by each respondent.

ii. Recoding of variables: The quantitative variables were recoded using different cut off points defined by ROC analysis to include them in the analysis and enable the estimation of risk.

iii. Computation of new variables like body mass index.

\section{B- Data analysis included:}

1- Chi squared test for the categorical variables

2- Fisher exact test when the expected cell count was less than 5 .

3- Calculation of the crude Odds Ratios to estimate the risk using the category with low breast cancer risk as a reference group.

4- Calculation of the mean $\pm S D$ for the quantitative variables.

5-Logistic Regression analysis was used to assess the impact of potential predictors obtained from patient history and clinical examination on the onset of breast cancer through the adjusted Odds Ratio. It is a powerful statistical tool for estimating the magnitude of the association between the exposure and a binary outcome (y), i.e, a variable with two possible values $(0,1)$. Y is the dependent variable.

$\mathrm{Y}=0$ for no cancer in histopathology.

$Y=1$ for cancer in histopathology.

$P$ value less than 0.05 was considered statistically significant.

\section{RESULTS}

The distributions of potential risk factors (RF) were presented in all subjects with and without breast cancer (table 1\&2). From the case-control comparisons, our results showed that age at presentation $\geq$ 60 years $(\mathrm{OR}=10.27,95 \% \mathrm{Cl}=3.44-30.6)$, delayed menopause $>50$ years $(\mathrm{OR}=5.23$, $95 \% \mathrm{Cl}=1.97-13.8)$, family history of breast cancer in first degree relatives $(\mathrm{OR}=10.5$, $95 \% \mathrm{Cl}=3.6-30.6)$ and oral contraceptive use $(\mathrm{OR}=2.57,95 \% \quad \mathrm{Cl}=1.12-5.89)$ were found to be significantly associated with 
higher risk of breast cancer in showed border line significance with 3.8 risk postmenopausal females $(\mathrm{p}<0.05)$.

(OR=3.8 for both) (table 4).

Body mass index (BMI) (OR=8.6, 95\%

Factors showing significant association $\mathrm{Cl}=2.04-36.2), \mathrm{WC}(\mathrm{OR}=2.85,95 \% \mathrm{Cl}=1.00$ with risk for breast cancer were additionally -8.17 ) and serum levels of estradiol $\geq 20 \mathrm{pg} / \mathrm{ml}$ analyzed in this study to investigate $(\mathrm{OR}=84.3,95 \% \mathrm{Cl}=22.3-319.2)$ were found whether or not they are risk factors for ERto be significantly associated with higher risk of breast cancer (Table 3).

positive and PR-positive breast carcinoma. These factors included age at presentation The independent effects of different risk $\geq 60$ years, age at menopause $>50$ years, factors after controlling the effect of other history of oral contraceptive use, body potential confounders were studied in a mass index $>40 \mathrm{~kg} / \mathrm{m}^{2}$, waist circumference logistic model. Age at presentation $\geq 60$ years, $\quad>100 \mathrm{~cm}$, total serum estradiol level $\geq 20$ late menopause $>50$ years and high total $\mathrm{pg} / \mathrm{ml}$ and family history of breast cancer in estradiol level $\geq 20 \mathrm{pg} / \mathrm{ml}$ were significant risk first-degree relatives. From the case-case factors $(\mathrm{OR}=13.2,95 \% \mathrm{Cl}=2.1-96.1 ; \mathrm{OR}=7.2$, comparisons, none of these factors $95 \% \mathrm{Cl}=2.16-24.48$ and $\mathrm{OR}=89.8,95 \% \mathrm{Cl}=$ showed significant association with the risk 25.9-323.2, respectively), while oral of developing ER-positive and PR-positive contraceptive use and increased WC $>100 \mathrm{~cm}$ breast carcinoma (tables 5\&6). 
Table (1): Crude odds ratios (ORs) calculated for age at presentation, menstrual factors, months of breast feeding and family history of breast cancer in first-degree relatives as risk factors for breast cancer among postmenopausal females.

\begin{tabular}{|c|c|c|c|c|c|c|c|c|}
\hline \multirow{2}{*}{\multicolumn{2}{|c|}{ Risk Factors }} & \multicolumn{2}{|c|}{$\begin{array}{c}\text { Breast cancer } \\
\text { group }\end{array}$} & \multicolumn{2}{|c|}{$\begin{array}{l}\begin{array}{l}\text { Control } \\
\text { group }\end{array} \\
\end{array}$} & \multicolumn{2}{|c|}{ Total } & \multirow[t]{2}{*}{$\begin{array}{l}P^{\star}(t) O^{\star \star} \\
(95 \% \mathrm{Cl})\end{array}$} \\
\hline & & No. & $\%$ & No. & $\%$ & No. & $\%$ & \\
\hline Age at presentation & $\begin{array}{l}<60 \text { yrs }{ }^{\circledR} \\
\geq 60 \text { yrs }\end{array}$ & $\begin{array}{l}35 \\
15\end{array}$ & $\begin{array}{l}41.2 \\
100\end{array}$ & $\begin{array}{c}50 \\
0\end{array}$ & $\begin{array}{c}58.8 \\
0.0\end{array}$ & $\begin{array}{l}85 \\
15\end{array}$ & $\begin{array}{l}100 \\
100\end{array}$ & $\begin{array}{c}0.000^{*} \\
10.27 \\
(3.44-30.6)\end{array}$ \\
\hline Age of menarche & $\begin{array}{c}\leq 12 \text { yrs } \\
>12 \text { yrs }{ }^{\circledR}\end{array}$ & $\begin{array}{l}18 \\
32\end{array}$ & $\begin{array}{l}54.5 \\
47.8\end{array}$ & $\begin{array}{l}15 \\
35\end{array}$ & $\begin{array}{l}45.5 \\
52.2\end{array}$ & $\begin{array}{l}33 \\
67\end{array}$ & $\begin{array}{l}100 \\
100\end{array}$ & $\begin{array}{c}0.52 \\
0.762 \\
(0.33-1.76)\end{array}$ \\
\hline Age at menopause & $\begin{array}{l}\leq 50 \text { yrs } \AA \\
>50 \text { yrs }\end{array}$ & $\begin{array}{c}7 \\
43\end{array}$ & $\begin{array}{l}23.3 \\
61.4\end{array}$ & $\begin{array}{l}23 \\
27\end{array}$ & $\begin{array}{l}76.7 \\
38.6\end{array}$ & $\begin{array}{l}30 \\
70\end{array}$ & $\begin{array}{l}100 \\
100\end{array}$ & $\begin{array}{c}0.000^{*} \\
5.23 \\
(1.97-13.8)\end{array}$ \\
\hline $\begin{array}{l}\text { Months of breast } \\
\text { feeding history of } \\
\text { Family histy } \\
\text { breast cancer in first- } \\
\text { degree relatives }\end{array}$ & $\begin{array}{c}\text { No } \\
\leq 24 \text { months } \\
>24 \text { months }{ }^{\circledR} \\
\text { No }{ }^{\circledR} \\
\text { Yes }\end{array}$ & $\begin{array}{c}7 \\
5 \\
38 \\
34 \\
16\end{array}$ & $\begin{array}{l}70 \\
62.5 \\
46.3 \\
40.5 \\
100\end{array}$ & $\begin{array}{c}3 \\
3 \\
44 \\
50 \\
0\end{array}$ & $\begin{array}{c}30 \\
37.5 \\
53.7 \\
59.5 \\
0.0\end{array}$ & $\begin{array}{c}10 \\
8 \\
82 \\
84 \\
16\end{array}$ & $\begin{array}{l}100 \\
100 \\
100 \\
100 \\
100\end{array}$ & $\begin{array}{c}0.28 \\
2.5(0.69-9.4) \\
1.9(0.45-8.05) \\
0.000^{*} \\
10.5 \\
(3.6-30.6)\end{array}$ \\
\hline
\end{tabular}

$P^{*}$ : Significance level at $5 \%(<0.05)$

(t) Chi-square test

$\mathrm{OR}^{\star *}$ : Non adjusted $\mathrm{OR}$ (crude OR): calculated for each patient characteristic.

(B) is the Reference group

Table (2): Crude odds ratios (ORs) calculated for reproductive and hypertension risk factors for postmenopausal breast cancer

\begin{tabular}{|c|c|c|c|c|c|c|c|c|}
\hline \multicolumn{2}{|l|}{ Risk Factors } & \multicolumn{2}{|c|}{$\begin{array}{c}\text { Breast } \\
\text { cancer group }\end{array}$} & \multicolumn{2}{|c|}{$\begin{array}{l}\text { Control } \\
\text { group }\end{array}$} & \multicolumn{2}{|c|}{ Total } & \multirow{2}{*}{$\begin{array}{c}P^{\star}(t) O R^{\star \star} \\
(95 \% \mathrm{Cl})\end{array}$} \\
\hline & & No. & $\%$ & No. & $\%$ & No. & $\%$ & \\
\hline \multirow{3}{*}{$\begin{array}{l}\text { Age at first full-term } \\
\text { pregnancy }\end{array}$} & $\leq 30$ yrs $\AA$ & 47 & 49.5 & 48 & 50.5 & 95 & 100 & 0.646 \\
\hline & $>30 \mathrm{yrs}$ & 3 & 60 & 2 & 40 & 5 & 100 & $\begin{array}{c}1.53 \\
(0.24-9.58)\end{array}$ \\
\hline & $\leq 2$ & 15 & 55.6 & 12 & 44.4 & 27 & 100 & 0.499 \\
\hline Parity & $>2 \AA$ & 35 & 47.9 & 38 & 52.1 & 73 & 100 & $\begin{array}{c}1.35 \\
(0.56-3.25)\end{array}$ \\
\hline \multirow[t]{2}{*}{ Abortion } & No $\AA^{\circledR}$ & 23 & 42.6 & 31 & 57.4 & 54 & 100 & 0.108 \\
\hline & Yes & 27 & 58.7 & 19 & 41.3 & 46 & 100 & $\begin{array}{c}1.9 \\
(0.86-4.25)\end{array}$ \\
\hline \multirow[t]{2}{*}{ Hypertension } & No $\AA_{8}$ & 30 & 49.2 & 31 & 50.8 & 61 & 100 & 0.83 \\
\hline & Yes & 20 & 51.3 & 19 & 48.7 & 39 & 100 & $\begin{array}{c}1.08 \\
(0.48-2.43)\end{array}$ \\
\hline \multirow{2}{*}{$\begin{array}{l}\text { Oral contraceptive use } \\
\text { before menopause }\end{array}$} & $\mathrm{No}{ }^{\circledR}$ & 14 & 35.9 & 25 & 64.1 & 39 & 100 & $0.02^{*}$ \\
\hline & Yes & 36 & 59 & 25 & 41 & 61 & 100 & $\begin{array}{c}2.57 \\
(1.12-5.89)\end{array}$ \\
\hline
\end{tabular}

$\mathrm{P}^{\star}$ : significance level at $5 \%(<0.05)$

(t) Chi-square test

$\mathrm{OR}^{* *}$ : non adjusted OR (crude OR): calculated for each patient characteristic.
(B) is the Reference group
(f) : Fisher exact test 
Table (3): Crude odds ratios (ORs) calculated for some obesity-related risk factors for postmenopausal breast cancer

\begin{tabular}{|c|c|c|c|c|c|c|c|c|}
\hline \multirow[t]{2}{*}{ Risk Factors } & & \multicolumn{2}{|c|}{$\begin{array}{c}\text { Breast } \\
\text { cancer group }\end{array}$} & \multicolumn{2}{|c|}{ Control group } & \multicolumn{2}{|c|}{ Total } & \multirow[t]{2}{*}{$\begin{array}{c}P^{\star}(t) O^{\star \star *} \\
(95 \% \mathrm{Cl})\end{array}$} \\
\hline & & No. & $\%$ & No. & $\%$ & No. & $\%$ & \\
\hline BMI & $(\leq 40) \AA$ & 42 & 45.7 & 50 & 54.3 & 92 & 100 & $0.006^{*}(f)$ \\
\hline$\left(\mathrm{Kg} / \mathrm{m}^{2}\right)$ & $(>40)$ & 8 & 100 & 0 & 0.0 & 8 & 100 & $\begin{array}{c}8.6 \\
(2.04-36.2)\end{array}$ \\
\hline Waist & $(\leq 100) \circledast$ & 6 & 30 & 14 & 70 & 20 & 100 & $0.046^{\star}$ \\
\hline $\begin{array}{l}\text { circumference } \\
(\mathrm{Cm})\end{array}$ & $(>100)$ & 44 & 55 & 36 & 72 & 80 & 100 & $\begin{array}{c}2.85 \\
(1.0-8.17)\end{array}$ \\
\hline Fasting blood & $\leq 110 \AA$ & 37 & 45.1 & 45 & 54.9 & 82 & 100 & $0.07(f)$ \\
\hline sugar (mg/dl) & $>110$ & 13 & 72.2 & 5 & 27.8 & 18 & 100 & $\begin{array}{c}4.26 \\
(0.81-22.53)\end{array}$ \\
\hline $\begin{array}{l}\text { Insulin level } \\
(\mu \mathrm{U} / \mathrm{ml})\end{array}$ & $\begin{array}{c}(\leq 4.9){ }^{\circledR} \\
(>4.9)\end{array}$ & $\begin{array}{l}11 \\
39\end{array}$ & $\begin{array}{l}37.9 \\
54.9\end{array}$ & $\begin{array}{l}18 \\
32\end{array}$ & $\begin{array}{l}62.1 \\
45.1\end{array}$ & $\begin{array}{l}29 \\
71\end{array}$ & $\begin{array}{l}100 \\
100\end{array}$ & $\begin{array}{c}0.123 \\
1.99 \\
(0.82-4.82)\end{array}$ \\
\hline Insulin & $(\leq 1.12) \circledast$ & 7 & 35 & 13 & 65 & 20 & 100 & 0.134 \\
\hline resistance & $(>1.12)$ & 43 & 53.8 & 37 & 46.2 & 80 & 100 & $\begin{array}{c}2.15 \\
(0.78-5.98)\end{array}$ \\
\hline $\begin{array}{c}\text { Serum total } \\
\text { estradiol level } \\
(\mathrm{pg} / \mathrm{ml})\end{array}$ & $\begin{array}{c}(<20){ }^{\circledR} \\
(\geq 20)\end{array}$ & $\begin{array}{c}4 \\
46\end{array}$ & $\begin{array}{c}8.3 \\
88.5\end{array}$ & $\begin{array}{c}44 \\
6\end{array}$ & $\begin{array}{l}91.7 \\
11.5\end{array}$ & $\begin{array}{l}48 \\
52\end{array}$ & $\begin{array}{l}100 \\
100\end{array}$ & $\begin{array}{c}0.000^{*} \\
84.3 \\
(22.3-319.17)\end{array}$ \\
\hline
\end{tabular}

$\mathrm{P}^{*}$ : significance level at $5 \%(<0.05)$

(t) Chi-square test

$\mathrm{OR}^{* *}$ : non adjusted OR (crude OR): calculated for each patient characteristic.
$\left({ }^{\circ}\right.$ is the Reference group
(f) : Fisher exact test

Table (4): Multivariate logistic regression analysis of risk factors for breast cancer among postmenopausal females

\begin{tabular}{|c|c|c|c|}
\hline Risk factors & P- value & $\mathrm{OR}^{(\mathrm{t})}$ & $95 \% \mathrm{Cl}$ \\
\hline $\begin{array}{l}\text { Family history of breast cancer } \\
\text { Yes vs } \mathrm{No}^{(\mathrm{tt})}\end{array}$ & 0.72 & 7.8 & $0.27-33.5$ \\
\hline $\begin{array}{l}\text { Age at presentation (years) } \\
\geq 60 \text { vs }<60^{(t)}\end{array}$ & $0.007^{*}$ & 13.2 & $2.1-96.1$ \\
\hline $\begin{array}{l}\text { Body mass index }\left(\mathrm{kg} / \mathrm{m}^{2}\right) \\
>40 \text { vs } \leq 40^{(\mathrm{tt})}\end{array}$ & 0.14 & 1.7 & $0.82-3.7$ \\
\hline $\begin{array}{l}\text { History of oral contraceptive use } \\
\text { Yes vs }^{\text {No(tt) }}\end{array}$ & 0.05 & 3.8 & $0.95-15.3$ \\
\hline $\begin{array}{l}\text { Age at menopause (years) } \\
>50 \text { vs } \leq 50^{(\mathrm{tt})}\end{array}$ & $0.008^{*}$ & 7.2 & $2.16-24.48$ \\
\hline $\begin{array}{l}\text { Total serum estradiol level (pg/ml) } \\
\geq 20 \text { vs }<20^{(\mathrm{tt})}\end{array}$ & $0.000^{*}$ & 89.8 & 25.9-323.2 \\
\hline $\begin{array}{l}\text { Waist circumference (cm) } \\
>100 \text { vs } \leq 100^{(\mathrm{tt})}\end{array}$ & 0.05 & 3.86 & $0.97-15.29$ \\
\hline
\end{tabular}

(t) Adjusted OR

(tt) Reference group

*significance was considered at P-value $<0.05$ 
Table (5): Crude odds ratios (ORs) calculated for selected risk factors according to ER status for breast cancer postmenopausal females

\begin{tabular}{|c|c|c|c|c|c|c|c|c|}
\hline \multirow[b]{2}{*}{ Risk Factors } & & \multicolumn{2}{|c|}{ ER-ve } & \multicolumn{2}{|c|}{ ER+ve } & \multicolumn{2}{|c|}{ Total } & \multirow{2}{*}{$\begin{array}{l}P^{\star}(t) O^{\star \star} \\
(95 \% C l)\end{array}$} \\
\hline & & No. & $\%$ & No. & $\%$ & No & $\%$ & \\
\hline Age at presentation & $\begin{array}{c}<60 \text { yrs } \AA \\
\geq 60 \text { yrs }\end{array}$ & $\begin{array}{c}11 \\
5\end{array}$ & $\begin{array}{l}31.4 \\
33.3\end{array}$ & $\begin{array}{l}24 \\
10\end{array}$ & $\begin{array}{l}68.6 \\
66.7\end{array}$ & $\begin{array}{l}35 \\
15\end{array}$ & $\begin{array}{l}100 \\
100\end{array}$ & $\begin{array}{c}0.572 \\
0.917 \\
(0.253-3.327)\end{array}$ \\
\hline Age at menopause & $\begin{array}{l}\leq 50 \text { yrs }{ }^{\circledR} \\
>50 \text { yrs }\end{array}$ & $\begin{array}{c}1 \\
15\end{array}$ & $\begin{array}{l}14.3 \\
34.9\end{array}$ & $\begin{array}{c}6 \\
28\end{array}$ & $\begin{array}{l}85.7 \\
65.1\end{array}$ & $\begin{array}{c}7 \\
43\end{array}$ & $\begin{array}{l}100 \\
100\end{array}$ & $\begin{array}{c}0.269 \\
0.311 \\
(0.034-2.830)\end{array}$ \\
\hline $\begin{array}{lr}\text { Oral contraceptive } \\
\text { use } & \text { before } \\
\text { menopause } & \end{array}$ & $\begin{array}{l}\text { No }{ }^{\circledR} \\
\text { Yes }\end{array}$ & $\begin{array}{c}4 \\
12\end{array}$ & $\begin{array}{l}28.6 \\
33.3\end{array}$ & $\begin{array}{l}10 \\
24\end{array}$ & $\begin{array}{l}71.4 \\
66.7\end{array}$ & $\begin{array}{l}14 \\
36\end{array}$ & $\begin{array}{l}100 \\
100\end{array}$ & $\begin{array}{c}0.513 \\
0.8 \\
(0.207-3.088)\end{array}$ \\
\hline $\begin{array}{l}\mathrm{BMI} \\
\left(\mathrm{Kg} / \mathrm{m}^{2}\right)\end{array}$ & $\begin{array}{c}(\leq 40) \circledast) \\
(>40)\end{array}$ & $\begin{array}{c}12 \\
4\end{array}$ & $\begin{array}{c}28.6 \\
50\end{array}$ & $\begin{array}{c}30 \\
4\end{array}$ & $\begin{array}{c}71.4 \\
50\end{array}$ & $\begin{array}{c}42 \\
8\end{array}$ & $\begin{array}{l}100 \\
100\end{array}$ & $\begin{array}{c}0.215 \\
0.4 \\
(0.086-1.364)\end{array}$ \\
\hline $\begin{array}{l}\text { Waist circumference } \\
(\mathrm{Cm})\end{array}$ & $\begin{array}{c}(\leq 100) \AA \\
(>100)\end{array}$ & $\begin{array}{c}2 \\
14\end{array}$ & $\begin{array}{l}33.3 \\
31.8\end{array}$ & $\begin{array}{c}4 \\
30\end{array}$ & $\begin{array}{l}66.7 \\
68.2\end{array}$ & $\begin{array}{c}6 \\
44\end{array}$ & $\begin{array}{l}100 \\
100\end{array}$ & $\begin{array}{c}0.635 \\
1.071 \\
(0.175-6.560)\end{array}$ \\
\hline $\begin{array}{l}\text { Serum total estradiol } \\
\text { level }(\mathrm{pg} / \mathrm{ml})\end{array}$ & $\begin{array}{l}(<20) \AA \\
(\geq 20)\end{array}$ & $\begin{array}{c}2 \\
14\end{array}$ & $\begin{array}{c}50 \\
30.4\end{array}$ & $\begin{array}{c}2 \\
32\end{array}$ & $\begin{array}{c}50 \\
69.6\end{array}$ & $\begin{array}{c}4 \\
46\end{array}$ & $\begin{array}{l}100 \\
100\end{array}$ & $\begin{array}{c}0.584 \\
2.286 \\
(0.292-17.9)\end{array}$ \\
\hline $\begin{array}{l}\text { Family history of } \\
\text { breast cancer in first- } \\
\text { degree relatives }\end{array}$ & $\begin{array}{l}\text { No }{ }^{\circledR} \\
\text { Yes }\end{array}$ & $\begin{array}{c}13 \\
3\end{array}$ & $\begin{array}{l}38.2 \\
18.8\end{array}$ & $\begin{array}{l}21 \\
13\end{array}$ & $\begin{array}{l}61.8 \\
81.3\end{array}$ & $\begin{array}{l}34 \\
16\end{array}$ & $\begin{array}{l}100 \\
100\end{array}$ & $\begin{array}{c}0.208 \\
2.683 \\
(0.64-11.247)\end{array}$ \\
\hline
\end{tabular}

ER: Estrogen receptor

$P^{*}$ : significance level at $5 \%(<0.05)$

(t) Chi-square test

$\mathrm{OR}^{\star *}$ : non adjusted OR (crude OR): calculated for each patient characteristic.

$(\AA$ is the Reference group

(f) : Fisher exact test 
Table (6): Crude odds ratios (ORs) calculated for selected risk factors according to PR status for breast cancer postmenopausal females

\begin{tabular}{|c|c|c|c|c|c|c|c|c|}
\hline \multirow{2}{*}{ Risk Factors } & & \multicolumn{2}{|c|}{ PR-ve } & \multicolumn{2}{|c|}{ PR+ve } & \multicolumn{2}{|c|}{$\overline{\text { Total }}$} & \multirow{2}{*}{$\begin{array}{c}P^{\star}(t) O^{*} R^{\star \star} \\
(95 \% \mathrm{Cl})\end{array}$} \\
\hline & & No. & $\%$ & No. & $\%$ & No & $\%$ & \\
\hline \multirow[t]{2}{*}{ Age at presentation } & $<60$ yrs ${ }^{\circledR}$ & 12 & 34.3 & 23 & 65.7 & 35 & 100 & 0.608 \\
\hline & $\geq 60$ yrs & 5 & 33.3 & 10 & 66.7 & 15 & 100 & $\begin{array}{c}1.043 \\
(0.290-3.755)\end{array}$ \\
\hline \multirow[t]{2}{*}{ Age at menopause } & $\leq \mathbf{5 0}$ yrs ${ }^{\circledR}$ & 2 & 28.6 & 5 & 71.4 & 7 & 100 & 0.544 \\
\hline & $>50$ yrs & 15 & 34.9 & 28 & 65.1 & 43 & 100 & $\begin{array}{c}0.747 \\
(0.129-4.321)\end{array}$ \\
\hline \multirow[t]{2}{*}{ Oral contraceptive use } & No $\AA^{\circledR}$ & 5 & 35.7 & 9 & 64.3 & 14 & 100 & 0.562 \\
\hline & Yes & 12 & 33.3 & 24 & 66.7 & 36 & 100 & $\begin{array}{c}1.111 \\
(0.305-4.054)\end{array}$ \\
\hline \multirow{2}{*}{$\begin{array}{l}\text { BMI } \\
\left(\mathrm{Kg} / \mathrm{m}^{2}\right)\end{array}$} & $(\leq 40) \circledast$ & 13 & 31 & 29 & 69 & 42 & 100 & 0.258 \\
\hline & $(>40)$ & 4 & 50 & 4 & 50 & 8 & 100 & $\begin{array}{c}(0.448 \\
(0.097-2.075)\end{array}$ \\
\hline \multirow{2}{*}{$\begin{array}{l}\text { Waist } \\
(\mathrm{Cm})\end{array}$} & $(\leq 100) \AA$ & 2 & 33.3 & 4 & 66.7 & 6 & 100 & 0.674 \\
\hline & $(>100)$ & 15 & 34.1 & 29 & 65.9 & 44 & 100 & $\begin{array}{c}0.967 \\
(0.158-5.896)\end{array}$ \\
\hline \multirow{2}{*}{$\begin{array}{l}\text { Serum total } \\
\text { level }(\mathrm{pg} / \mathrm{ml})\end{array}$} & $(<20) \AA$ & 2 & 50 & 2 & 50 & 4 & 100 & 0.420 \\
\hline & $(\geq 20)$ & 15 & 32.6 & 31 & 67.4 & 46 & 100 & $\begin{array}{c}2.067 \\
(0.265-16.128)\end{array}$ \\
\hline \multirow{2}{*}{$\begin{array}{l}\text { Family history of breast } \\
\text { cancer in first degree } \\
\text { relatives }\end{array}$} & No $\AA$ & 14 & 41.2 & 20 & 58.8 & 34 & 100 & 0.106 \\
\hline & Yes & 3 & 18.8 & 13 & 81.3 & 16 & 100 & $\begin{array}{c}3.033 \\
(0.727-12.665)\end{array}$ \\
\hline $\begin{array}{l}\text { PR: Progesterone recepto } \\
\mathrm{P}^{\star}: \text { significance level at } 5 \% \\
\text { (t) Chi-square test } \\
\text { OR }^{\star *}: \text { non adjusted OR (C) } \\
\text { (is the Reference group } \\
\text { (f) : Fisher exact test }\end{array}$ & $(<0.05)$ & & & & & & & \\
\hline
\end{tabular}

\section{DISCUSSION}

With respect to the significant association between age at presentation and the risk of postmenopausal breast cancer, the explanation of this result may be as age increases, body adiposity increases, with increase in the activity of aromatase enzyme in adipocytes which catalyzes the conversion of androgens to estrogens with the induction of breast carcinoma. Ries et al. ${ }^{(15)}$ reported that as age increases, the risk for breast cancer increases in postmenopausal women. Concerning the effects of lactation, ages at menarche and menopause on postmenopausal breast cancer risk, our 
results were in concordant with those of Kawai et al. ${ }^{(16)}$

Regarding the association between family history of breast cancer in first degree relatives and the risk of postmenopausal breast cancer, it was mentioned that breast cancer tends to cluster in families. Women with a family history of breast cancer, particularly in a first-degree relative, have approximately double the risk of developing breast cancer compared to women without such a history. ${ }^{(17)}$ Gene linkage studies pointed to loci on chromosomes 13 and 17 and cloning identified two genes, BRCA1 (on chromosome 17) and BRCA2 (on chromosome 13), that appear to be associated with the majority of inherited breast cancers, which account for $2-5 \%$ of all breast cancers. ${ }^{(18)}$

The results of the present study showed that age at first full-term pregnancy and parity were non-significantly associated, while oral contraceptive use was significantly associated with the risk of breast cancer in postmenopausal females. The proliferative effects of endogenous hormones support observations that exogenous exposure to hormones, primarily estrogen and progestin, is associated with breast cancer risk. Oral contraceptives provide a steady low level of hormones, which may be higher and more consistent than those occurring naturally. ${ }^{(19)}$ It was found that estradiol is a primary stimulant for breast cells proliferation. ${ }^{(20)}$ The simultaneous presence of progesterone may increase the rate of proliferation. ${ }^{(21)}$ This conclusion is based on the fact that breast mitotic activity peaks during the luteal phase of the menstrual cycle. ${ }^{(22)}$ Our results were in agreement with those of Phipps et al. ${ }^{(23)}$

For the effect of abortion, high glucose levels and hypertension, our results were in agreement with Veronesi et al. ${ }^{(24)}$, Kabat et al. ${ }^{(25)}$ and Peeters et al. ${ }^{(26)}$, respectively.

The results of the current study showed that increased BMI, WC, and serum level of total estradiol were significantly associated with breast cancer risk in 
postmenopusal females which were in agreement with the results found by McTiernan et al. ${ }^{(27)}$ and Friedenreich. ${ }^{(28)}$ Friedenreich. ${ }^{(28)}$ postulated that obesity may increase levels of circulating endogenous sex hormones which, in turn, increase breast cancer risk. Also, he suggested that with obesity, there are increased levels of fat tissue that can store toxins and can serve as a continuous source of carcinogens. ${ }^{(28)}$

Although, Kabat et $\mathrm{al}^{(25)}$ suggested that elevated serum insulin levels may be a risk factor for postmenopausal breast cancer, our results showed non-significant association between serum insulin and insulin resistance among postmenopausal breast cancer females.

\section{Multivariate Logistic regression analysis} with the risk for breast cancer was the dependent variable and family history of breast cancer in first-degree relatives, age at presentation $\geq 60$ yaers, BMl $>40 \mathrm{~kg} / \mathrm{m}^{2}$, history of oral contraceptive use before menopause, age at menopause $>50$ years, WC $>100 \mathrm{~cm}$ and serum total estradiol levels $\geq 20 \mathrm{pg} / \mathrm{ml}$ were independent variables, showed that the variables including age at presentation $\geq 60$ years, age at menopause > 50 years and serum total estradiol levels $\geq 20$ $\mathrm{pg} / \mathrm{ml}$ were the strongest risk factors for breast cancer in postmenopausal females. This means that postmenopausal females should continuously do breast self- examination for their breast monthly and do their best to prevent themselves from getting obese by maintaining physical exercise and decrease calories intake in their diets in order to protect themselves from getting breast carcinoma.

Regarding the absence of the association between the studied factors and the risk for ER-positive and PR-positive breast cancer, our results are in agreement with many studies. Rosenberg et al. ${ }^{(29)}$ found nonsignificant association of age at menopause and oral contraceptive use before menopause with the risk of developing ER- positive and PR-positive breast tumors in postmenopausal 
females. Pinheiro et al. ${ }^{(30)}$ demonstrated nonsignificant association of BMI and waist circumference with the hormonal receptor status in breast tumors of postmenopausal females. Sieri et al. ${ }^{(31)}$ reported no significant association between serum level of total estradiol and the risk of devepoing ERpositive and PR- positive breast tumors in postmenopausal females. Althuis et al. ${ }^{(4)}$ found that risks associated with family history of breast cancer in first-degree relatives did not differ by receptor status of breast tumors among postmenopausal females. This means that all of these risk factors are similarly related to breast cancer regardless of hormonal receptor status. Yoo et al. ${ }^{(32)}$ reported that age at presentation was a significant risk factor for PR-positive but not ER-positive breast tumors in postmenopausal females.

\section{REFERENCES}

1. Pichard C, Plu-Bureau G, Neves E, Castro M, Gompel A. Insulin resistance, obesity and breast cancer risk. Maturitas 2008; 60:19-30.

2. Vona-Davis L, Howard-McNatt $M$,
Rose DP. Complications of obesity: Adiposity, type-2 diabetes and the metabolic syndrome in breast cancer. Obesity Reviews 2007; 8: 395-408.

3. Conover CA, Lee PD, Kanaley JA, Clarkson JT, Jensen MD. Insulin regulation of insulin-like growth factor binding protein- 1 in obese and nonobese humans. J Clin Endocrinol Metab 2009; 74:1355-60.

4. Althuis MD, Fergenbaum JH, GarciaClosas M, Brinton LA, Madigan MP, Sherman ME. Etiology of hormone receptor defined breast cancer: A systematic review of the literature. Cancer Epidemiol Biomarkers Prev 2004;13:1558-68.

5. Manjer J, Malina J, Berglund G, Bondeson L, Garne JP, Janzon L. Smoking associated with hormone receptor negative breast cancer. Int $\mathrm{J}$ Cancer 2001; 91: 580-4.

6. Chen WY, Colditz GA. Risk factors and hormone-receptor status: Epidemiology, risk-prediction models and treatment implications for breast cancer. Nat Clin Pract Oncol 2007; 4:415-23.

7. Terry MB, Zhang FF, Kabat G, Britton JA, Teitelbaum SL, Neugut Al, et al. Lifetime alcohol intake and breast cancer risk. Ann Epidemiol 2006; 16: 230-40.

8. Iwasaki M, Otan T, Inoue M, Sasazuki S, Tsugane S. Body size and risk for breast cancer in relation to estrogen and progesterone receptor status in Japan. Ann Epidemiol 2007; 17:30412.

9. Huang WY, Newman B, Millikan RC, Schell MJ, Hulka BS, Moorman PG. Hormone-related factors and risk of breast cancer in relation to estrogen receptor and progesterone receptor status. Am J Epidemiol 2000; 151: 703-14.

10. $\mathrm{Li} \mathrm{Cl}$, Malone KE, Daling JR. 
Interactions between body mass index and hormone therapy and postmenopausal breast cancer risk (United States). Cancer Causes Control 2006; 17:695-703.

11. Dey S, Soliman AS, Hablas A, Seifeldin IA, Ismail K, Ramadan M, et al. Urban-rural differences in breast cancer incidence by hormone receptor status across 6 years in Egypt. Breast Cancer Res Treat 2010; 120: 149-60.

12. Hintze J. NCSS (Number Cruncher Statistical System): Statistical Software, PASS (Power Analysis and Sample Size). Kaysville, Utah 2000.

13. Burits CA, Ashwood ER. Toetz textbook of clinical chemistry; $3^{\text {rd }}$ ed. WB Saunders Company. Philadelphia. 1999; pp 778-9.

14. Bonora E, Targher G, Alberiche M, Bonadonna RC, Saggiani F, Zenere $\mathrm{MB}$, et al. Homeostasis Model Assessment: Closely mirrors the glucose clamp technique in the assessment of insulin sensitivity. Diabetes Care 2000; 23: 57-63.

15. Ries LAG, Melbert D, Krapcho M, Mariotto A, Miller BA, Feuer EJ, et al. SEER Cancer Statistics Review, 1975-2004, National Cancer Institute. Bethesda, $\mathrm{MD}$, http://seer.cancer.gov/csr/1975_2004/, based on November 2006 SEER data submission, posted to the SEER web site, 2007.

16. Kawai M, Minami $Y$, Kuriyama S, Kakizaki M, Kakugawa Y, Nishino Y, et al. Reproductive factors, exogenous female hormone use and breast cancer risk in Japanese: The Miyagi Cohort Study. Cancer Causes Control 2010; 21:135-45.

17. Collaborative Group. Familial breast cancer: Collaborative reanalysis of individual data from 52 epidemiological studies including 58,209 women with breast cancer and
101,986 women without the disease. Lancet 2001;358: 1389-99.

18. Easton D, Ford D, Peto J. Inherited susceptibility to breast cancer. Cancer Surv 1993; 18:95-113.

19. Bernstein L. Epidemiology of endocrine-related risk factors for breast cancer. J Mammary Gland Biol Neoplasia 2002; 7: 3-15.

20. Henderson BE, Ross RK, Bernstein I. Estrogens as a cause of human cancer: The Richard and Hinda Rosenthal foundation award lecture. Cancer Res 1988; 48: 246-53.

21. Key TJA, Pike MC. The role of estrogens and progesterons in the epidemiology and prevention of breast cancer. EUR J Cancer Clin Oncol 1988; 24: 29-43.

22. Ferguson DJP, Anderson TJ. Morphological evaluation of cell turnover in relation to the menstrual cycle in the "resting" human breast. Br J Cancer 1981; 44:177-81.

23. Phipps Al, Malone KE, Porter PL, Daling JR, Li Cl. Reproductive and hormonal risk factors for postmenopausal luminal, HER2overexpressing, and triple-negative breast cancer. Cancer 2008; 113: 1521-6.

24. Veronesi U, Boyle P, Goldhirsch A, Orecchia R, Viale G. Breast cancer. Lancet 2005; 365: 1727-41.

25. Kabat GC, Kim M, Caan BJ, Chlebowski RT, Gunter MJ, Ho GY, et al. Repeated measures of serum glucose and insulin in relation to postmenopausal breast cancer. Int $\mathrm{J}$ Cancer 2009; 125, 2704-10.

26. Peeters PH, van Noord PA, Hoes AW, Fracheboud J, Gimbrère $\mathrm{CH}$, Grobbee DE. Hypertension and breast cancer risk in a 19-year follow-up study (the DOM cohort). Diagnostic investigation into mammalian cancer. J Hypertens 2000; 18:249-54. 
27. McTiernan A, Rajan KB, Tworoger SS, Irwin M, Bernstein L, Baumgartner R, et al. Adiposity and sex hormones in postmenopausal breast cancer survivors. Journal of Clinical Oncology 2003; 21: 1961-6.

28. Friedenreich CM. Review of anthropometric factors and breast cancer risk. Eur J Cancer Prev 2001; 10:15-32.

29. Rosenberg LU, Einarsdottir K, Friman EI, Wedren S, Dickman PW, Hall P, et al. Risk factors for hormone receptordefined breast cancer in postmenopausal women. Cancer Epidemiol Biomarkers Prev 2006; 15:2482-8.

30. Pinheiro RL, Sarian LO, Pinto-Neto AM, Morais S, Costa-Paiva L.
Relationship between body mass index, waist circumference and waist to hip ratio and the steroid hormone receptor status in breast carcinoma of pre- and postmenopausal women. The Breast 2009; 18: 8-12.

31. Sieri S, Krogh V, Bolelli G, Abagnato CA, Grioni S, Pala V, et al. Sex hormone levels, breast cancer risk, and cancer receptor status in postmenopausal women: The ORDET cohort. Cancer Epidemiol Biomarkers Prev. 2009;18:169-76.

32. Yoo K, Tajima K, Miura S, Takeuchi T, Hirose K, Risch H, et al. Breast cancer risk factors according to combined estrogen and progesterone receptor status: A case-control analysis. Am J Epidemiol 1997; 146: 307-14. 